\title{
MiR-206 Controls the Phenotypic Modulation of Pulmonary Arterial Smooth Muscle Cells Induced by Serum from Rats with Hepatopulmonary Syndrome by Regulating the Target Gene, Annexin A2
}

\author{
Lin Chen ${ }^{a}$ Yong-shuai Li ${ }^{a}$ Jian Cuia Jiao-nin Ning ${ }^{a}$ Guan-song Wang ${ }^{b}$ \\ Gui-sheng Qian $^{b}$ Kai-zhi Lu ${ }^{a}$ Bin Yia,b
}

aDepartment of Anesthesia, Southwest Hospital, The Third Military Medical University, Chongqing, 'Institute of Respiratory Disease, Xinqiao Hospital, The Third Military Medical University, Chongqing, China

\section{Key Words}

MicroRNA • ANXA2 • PASMCs • Phenotype modulation

\begin{abstract}
Background: Hepatopulmonary syndrome (HPS) is a serious complication of advanced liver disease that is characterised by intrapulmonary vascular dilatation (IPVD) and arterial hypoxemia. Pulmonary vascular remodelling (PVR) is an important pathological feature of HPS, but the potential mechanisms underlying PVR remain undefined. Recent findings have established the essential role of changes in Annexin A2 (ANXA2) in controlling the phenotypic modulation of pulmonary artery smooth muscle cells (PASMCs) in PVR associated with HPS. However, the mechanism by which upstream signalling regulates ANXA2 is unclear. Methods: In the present study, computational analysis was used to predict which miRNA might target the $3^{\prime}$-untranslated region ( $3^{\prime}$-UTR) of the ANXA2 mRNA. Real-time PCR and western blotting were performed to study the level of correlation between ANXA2 and the differentiation marker with the predicted miRNAs in PASMCs stimulated with serum from normal rats or those with HPS. Functional analysis of the miRNA and a luciferase reporter assay were performed to demonstrate that the predicted miRNA suppressed ANXA2 expression by directly targeting the predicted 3'-UTR site of the ANXA2 mRNA. Results: Computational analysis predicted that miR-206 would target the 3'-UTR of ANXA2 mRNA. In HPS rat serum-stimulated PASMCs, the expression of miR-206 displayed an inverse correlation with ANXA2, while a positive correlation was observed with the phenotypic marker smooth muscle $\alpha$-actin (SM $\alpha$-actin). The miRNA functional analysis and luciferase reporter assay demonstrated that miR-206 effectively downregulated the expression of ANXA2 by binding to the 3'-UTR of the ANXA2 mRNA. Consistently, miR-206 effectively inhibited the HPS rat serum-induced phenotypic


modulation and proliferation, while these effects were reversed in ANXA2-overexpressing PASMCs. Conclusion: This study demonstrates that miR-206 inhibits the HPS rat seruminduced phenotypic modulation and proliferation in PASMCs by down-regulating ANXA2 gene expression.

Copyright (C) 2014 S. Karger AG, Basel

\section{Introduction}

Hepatopulmonary syndrome (HPS) is a serious complication of advanced liver disease characterised by intrapulmonary vascular dilatation (IPVD) and arterial hypoxemia. The reported incidence of HPS varies from $4 \%$ to $47 \%$ due to non-uniform diagnostic criteria, and the 2.5-year mortality rate following diagnosis with HPS reaches $41 \%$ [1]. There is currently no effective pharmacological therapy for HPS, and orthotopic liver transplantation is considered to be the only reliable treatment. The main reason for the lack of viable therapeutic measures is that the exact pathogenesis of HPS has not been identified.

Previous studies and our initial research have demonstrated that pulmonary vascular remodelling (PVR) may play an important role in the pathogenesis of HPS. In the pathogenesis of PVR, pulmonary artery smooth muscle cells (PASMCs) switch from a quiescent and contractile phenotype to a proliferative and synthetic phenotype, while endothelial cells differentiate into smooth muscle-like cells. These two features constitute the two basic pathophysiological components [2]. The identification of molecular mechanisms underlying the phenotypic modulation of PASMCs could facilitate the development of improved treatments for HPS.

Annexin A2 (ANXA2), also known as lipocortin II or p36, is a 36-kDa protein member of the annexin superfamily of calcium-dependent phospholipid-binding proteins [3]. The ability of ANXA2 to form a heterotetrameric complex plays a key role in $\mathrm{Ca}^{2+}$-dependent exocytosis, endocytosis and membrane organisation [4-6]. Previous studies have indicated that ANXA2 is an important regulatory protein in the process of proliferation, migration, skeletal formation and angiogenesis in a variety of malignant tumours, such as liver cancer, pancreatic cancer, breast cancer and lung cancer [7-9]. We have recently demonstrated that ANXA2 plays an important role in the process of PASMC phenotypic switching [10]. However, little is known regarding the regulation of ANXA2 gene expression. Previous work has shown that the expression of ANXA2 mRNA can be positively or negatively regulated by stimulation with Hypoxia-Inducible Factor 1 or prostaglandin E2, respectively $[11,12]$. Recently, we also demonstrated that ANXA2 is regulated by serum from HPS rats. However, additional factors might be expected to restrict and tightly regulate the gene expression of ANXA2 in PASMCs.

MicroRNAs (miRNAs) are small noncoding RNAs that post-transcriptionally regulate the expression of the genes that control multiple biological processes [13]. In PASMCs, the stagespecific expression of certain miRNAs suggests their participation in PASMC phenotypic switching, which might contribute to the pathogenesis of PVR [14]. Therefore, we investigated whether some potential miRNAs implicated in PASMC phenotypic switching might regulate ANXA2 gene expression. In this study, we demonstrate that miR-206 directly downregulates ANXA2 gene expression by binding to the $3^{\prime}$-UTR of its mRNA, thus modulating the effects of this protein on phenotypic switching and proliferation by the PASMCs. These findings identify a role for miR-206 in the control of PASMC biology.

\section{Materials and Methods}

\section{Reagents and antibodies}

The reagents used in this study included rno-miR-206 mimics, inhibitors and negative controls, miRNA Isolation Kits, miScript II RT Kits, miScript SYBR Green PCR miRNA Detection Kits, and real-time PCR primers (Qiagen, USA); real-time RT-PCR assay kits (Takara, China); Fast-Plus EvaGreen master mix(Biotium, USA); rabbit anti-rat ANXA2 antibody, rabbit anti-rat $\beta$-actin antibody, mouse anti-rat SM $\alpha$-actin antibody, HRP- 
conjugated goat anti-mouse IgG and HRP-conjugated goat anti-rabbit IgG(RD Systems, USA); Alexa Fluor 488 goat anti-mouse IgG antibody and Alexa Fluor 555 goat anti-rabbit IgG (Earthox,USA); SDS-PAGE gel kits, mammalian protein extraction kits, BCA protein assay kits and eECL western blot kits (CWBIO, China); Lipofectamine 2000 (Invitrogen,USA); and CCK-8 (Dojindo Laboratories, Japan) (Sigma-Aldrich, USA). ${ }^{3} \mathrm{H}-\mathrm{TdR}$ was given as a generous gift from the Department of Nuclear Medicine, Southwest Hospital, The Third Military Medical University.

\section{A search for miRNA target genes and its binding site}

Three miRNA target prediction algorithms, PicTar (http://pictar.mdc-berlin.de), miRanda (http:// www.microrna.org) and TargetScan (http://www.targetscan.org), were used to identify the miRNAs possibly implicated in the regulation of ANXA2 gene expression. Subsequently, the same programs were used to identify the potential miRNA target genes. The miRanda algorithm was used to predict the putative miRNA binding sites.

\section{Cell culture}

The rat PASMCs were isolated from rat pulmonary arteries as previously described [10]. Briefly, healthy Sprague-Dawley rats were killed by exsanguination under pentothal anaesthesia. After the adventitia and endothelia were removed, the pulmonary artery was cut into small pieces and then cultured using the tissue-sticking method [15]. The purity and identity of the PASMCs was verified by typical morphological patterns and immunocytochemical staining using specific monoclonal antibodies raised against SM $\alpha$-actin, as previously reported $[16,17]$. The PASMCs were used for experiments between passages 5 and 9 . Cultures of PASMCs at a density of $10^{6} / \mathrm{cm}^{2}$ were divided into two groups: the control group, consisting of PASMCs that were cultured in DMEM supplemented with 5\% normal rat serum,and the HPS group, consisting of PASMCs that were incubated in DMEM containing 5\% serum from the HPS ratsfor various time periods (24 $\mathrm{h}, 48 \mathrm{~h}$ and $72 \mathrm{~h})$.

\section{HPS rat model}

All procedures performed on the rats were conducted according to the guidelines from the National Institutes of Health. The study's protocol was approved by the Committee on Animal Research of Southwest Hospital. The HPS rat model was established as described in our previous study [18]. Briefly, HPS was induced in Sprague-Dawley rats (180-220 g) by common bile duct ligation (CBDL). HPS rat serum was obtained from the CBDL rats that met the following criteria: 1) gas exchange abnormalities $(\mathrm{PaO} 2<85$ $\mathrm{mmHg}$ and $\mathrm{P}(\mathrm{A}-\mathrm{a}) 02>18 \mathrm{mmHg}$ ) and 2) intrapulmonary vascular dilation, confirmed by histopathological analysis. Normal rat serum was obtained from the rats in the sham-operated control group. All of the serum samples were stored at $-80^{\circ} \mathrm{C}$ for use in the subsequent experiments.

\section{Transfection}

After growth arrest, PASMCs were transfected with different groups of oligo nucleotides. The transfection complexes were prepared according to the manufacturer's instructions. For the overexpression of miR-206, an miR-206 mimic(10 nmol/L) was added to the complexes. Alternatively, an miR-206 inhibitor $(200 \mathrm{nmol} / \mathrm{L})$ was added to the complexes to induce knockdownof miR-206. The overexpression of ANXA2 was achieved by transient transfection using an ANXA2-expressing plasmid. The controls consisted of the application of oligo controls for the miR-206 mimic or miR-206 inhibitor, or an empty plasmid. At $24 \mathrm{~h}$ prior to treatment, miR-206, ANXA2 or both were transfected into the PASMCs using the Lipofectamine2000 reagent. The efficiency of transfection was confirmed using real-time PCR and western blotting.

\section{Western blotting}

The cultured cells were lysed and subjected to total protein extraction, according to the manufacturer's instructions. After the protein concentrations were determined using the BCA protein assay, equal quantities of protein were analysed by sodium dodecyl sulphate polyacrylamide gel electrophoresis (SDS-PAGE) and then transferred onto the PVDF membranes. The membranes were blocked for $1 \mathrm{~h}$ using the blocking solution and then incubated overnight with the primary antibody (1:1000 dilution) at $4^{\circ} \mathrm{C}$. After washing three times with Tris-Buffered Saline Tween-20 (TBST), the membrane was incubated with HRP-conjugated rabbit anti-goat IgG at a 1:1000 dilution for $1 \mathrm{~h}$ at room temperature before being washed again in TBST and 
visualised with an enhanced chemiluminescence reagent. Finally, the immunoreactive bands were scanned and stored using a gel imaging system. The intensity of the immunoreactivity was measured and analysed with an Alpha Imager.

\section{Real-time PCR}

Total RNA was extracted using the miRNeasy isolation kit. For the measurement of ANXA2 mRNA expression, the RNA $(1 \mu \mathrm{g})$ was reverse-transcribed using the real-time PCR assay kits, followed by realtime PCR using the Fast-Plus EvaGreen master mix. For the miRNA expression analysis, the RNA $(2 \mu \mathrm{g})$ was reverse-transcribed using the miScript II RT Kit, followed by real time PCR using the miScript SYBR Green PCR miRNA Detection Kit. The amplification and detection of the specific products were performed using the iCycleriQ Real-Time PCR Detection System. U6 was used as an internal control for the miRNA template normalisation, and glyceraldehyde 3-phosphate dehydrogenase (GADPH) was used for the other template normalisations. The PCR primers for ANXA2 were as follows: forward, 5'-TGAGCGGGATGCTTTGAAC-3', and reverse, 5'-ATCCTGTCTC TGTGCATTGCTG-3'.The GADPH PCR primers were as follows: forward, 5'GGCACAGTCAAGGCTGAGAATG-3', and reverse, 5' - ATGGTGGTGAAGACGCCAGTA -3'.

\section{Immunofluorescence}

For immunofluorescence, the PASMCs were fixed with 4\% paraformaldehyde and permeabilised with $0.2 \%$ Triton X-100. After blocking with 1\% goat serum, the PASMCs were incubated with a 1:100 dilution of the ANXA2 antibody and a 1:100 dilution of the SM $\alpha$-actin antibody at $4^{\circ}$ Covernight. Following washing, the sections were incubated with Alexa Fluor 488 goat anti-mouse IgG antibody and Alexa Fluor 555 rabbit anti-goat IgG antibodies for $2 \mathrm{~h}$ at room temperature. The nuclear DNA was stained with DAPI. The images were then captured using a Leica DM 3000 microscope and photographed using a DFC420 camera.

\section{Luciferase assay}

HEK 293 cells were co-transfected with the constitutively active Renillareniformis luciferaseproducing vector pRL, the hsa-miR-206 or a non-targeting pre-miR-negative control and the luciferase wild-type or mutated 3'-UTR vectors for ANXA2 using the SiportNeoFX transfection reagent according to the manufacturer's instructions. At 20 hours after the transfection, the cells were lysed, and the relative luciferase expression was measured on a scintillation counter with a dual luciferase reporter system.

\section{Thymidine incorporation assay}

Equal numbers of PASMCs were seeded into the wells of a 96-well plate in complete medium. After $24 \mathrm{~h}$ of synchronous growth, the cells were transfected with the miR-206 mimic, ANXA2 or both according to the manufacturer's instructions. Next, the cells were incubated with DMEM containing the different sera for the indicated periods, and the medium was supplemented with $1 \mu \mathrm{Ci}{ }^{3} \mathrm{H}-\mathrm{TdR}$ during the last $6 \mathrm{~h}$ of the treatment period. The incorporation was stopped with cold PBS solution, and $0.25 \%$ trypsin was added to separate the cells from the cell wall. The cells were collected onto a glass fibre filter with a multi-head harvester. Then, the cells were washed 3 times with physiological saline, stabilised with $10 \%$ trichloroacetic acid, decolourised with absolute ethanol, dried for $30 \mathrm{~min}$ at $80^{\circ} \mathrm{C}$, transferred into scintillation fluid and counted with a liquid scintillation counter (counts/min, cpm).

\section{CCK-8 assay}

The cellular proliferation was measuredby the Cell Counting Kit-8 (CСK-8) assay. Accordingly, $24 \mathrm{~h}$ after seeding equal numbers of PASMCs, the cells were transfected with the miR-206 mimic, ANXA2 or both. After transfection, the cells were incubated with DMEM containing the different sera for the indicated time periods. At the end of treatment, $10 \mu \mathrm{l}$ of the CCK-8 solution was added to each well, and cells were cultured for $2 \mathrm{~h}$. After that, the numbers of viable cells were quantified by measuring the absorbance value at $450 \mathrm{~nm}$ using a microplate absorbance reader.

\section{Statistical analysis}

All data are expressed as the mean \pm SEM. The comparisons between groups were carried out using Student's t-test or a Mann-Whitney U-test for parametric or non-parametric data, respectively. The results were considered to be statistically significant when $\mathrm{p}<0.05$. 


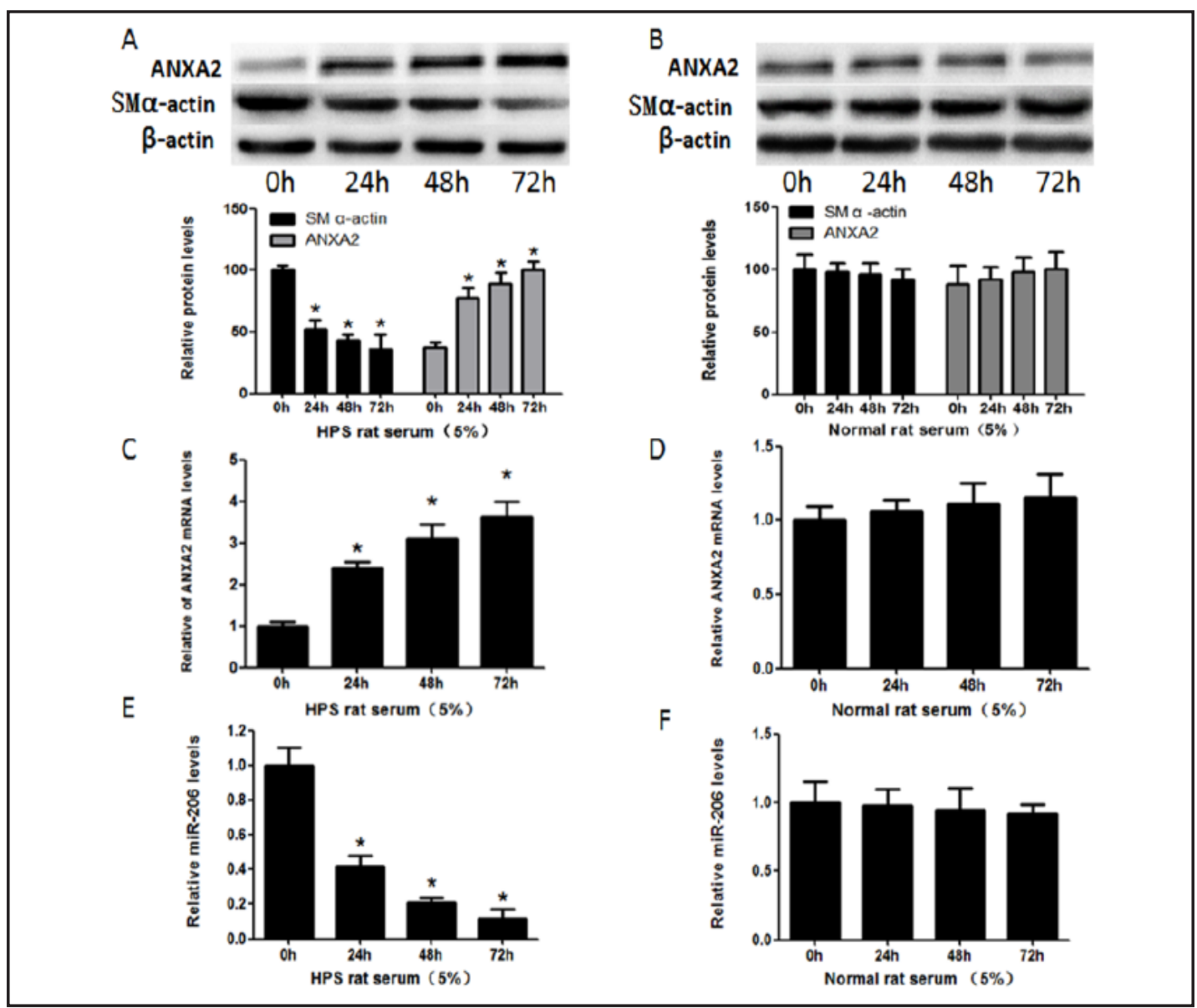

Fig. 1. Effect of HPS rat serum on the expression levels of miR-206 and ANXA2, which exhibited a negative relationship in cultured PASMCs. (A) Expression levels of ANXA2 and the PASMC phenotype marker SM $\alpha$-actin in PASMCs stimulated with HPS rat serum $24 \mathrm{~h}$ after the serum withdrawal. Representative western blot bands (top) and the mean data generated by densitometry analysis (bottom). The data shown are the mean \pm SEM; $n=4$. ${ }^{*} \mathrm{P}<0.05$ compared with the 0 h group. (B) Expression levels of ANXA2 and PASMC phenotype marker proteins in PASMCs stimulated with normal rat serum $24 \mathrm{~h}$ after the serum withdrawal. Representative western blot bands (top) and mean data generated by densitometry analysis (bottom). The data shown are the mean \pm SEM; $n=4$. ${ }^{*} \mathrm{P}<0.05$ compared with the $0 \mathrm{~h}$ group. (C) HPS rat serum had a timedependent effect on the ANXA2 mRNA expression during the $48 \mathrm{~h}$ following $24 \mathrm{~h}$ of serum withdrawal, as determined by real-time PCR. The data shown are the mean \pm SEM; $n=4$. ${ }^{*} \mathrm{P}<0.05$ compared with vehicle control. (D) ANXA2 mRNA levels in normal rat serum-stimulated PASMCs, determined by real-time PCR. The data shown are the mean $\pm \mathrm{SEM} ; \mathrm{n}=4$. ${ }^{*} \mathrm{P}<0.05$ compared with the 0 h group. (E) HPS rat serum caused a timedependent decrease in miR-206 expression $24 \mathrm{~h}$ after the serum withdrawal, as determined by real-time PCR. The data shown are the mean \pm SEM; $n=4$. ${ }^{*} \mathrm{P}<0.05$ compared with the 0 h group. (F) Normal rat serum caused no significant changes in miR-206 expression, as determined by real-time PCR. The data shown are the mean \pm SEM; $n=4$. ${ }^{*} \mathrm{P}<0.05$ compared with the 0 h group.

\section{Results}

Correlation of miR-206 with ANXA2 and the differentiation marker in PASMCs

According to the results of a previous computational analysis, three miRNAs were simultaneously predicted to target the 3'-UTR of the ANXA2 mRNA by PicTar, miRanda and TargetScan. Of these three, miR-206 was the only miRNA found to be downregulated in the cultured PASMCs. Therefore, we investigated the potential correlation of the expression 
A

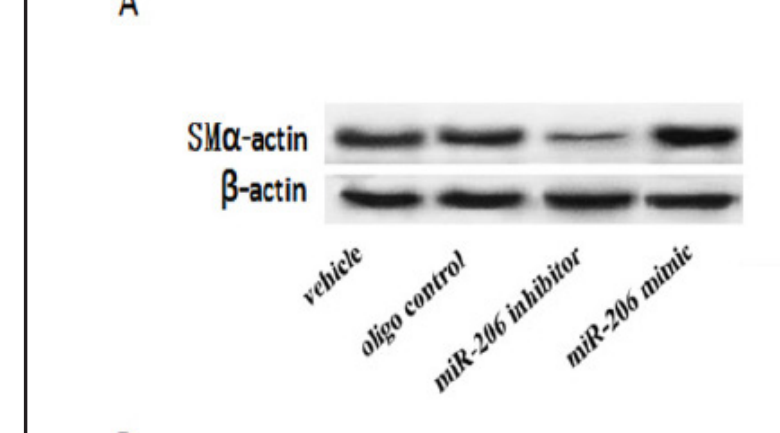

B
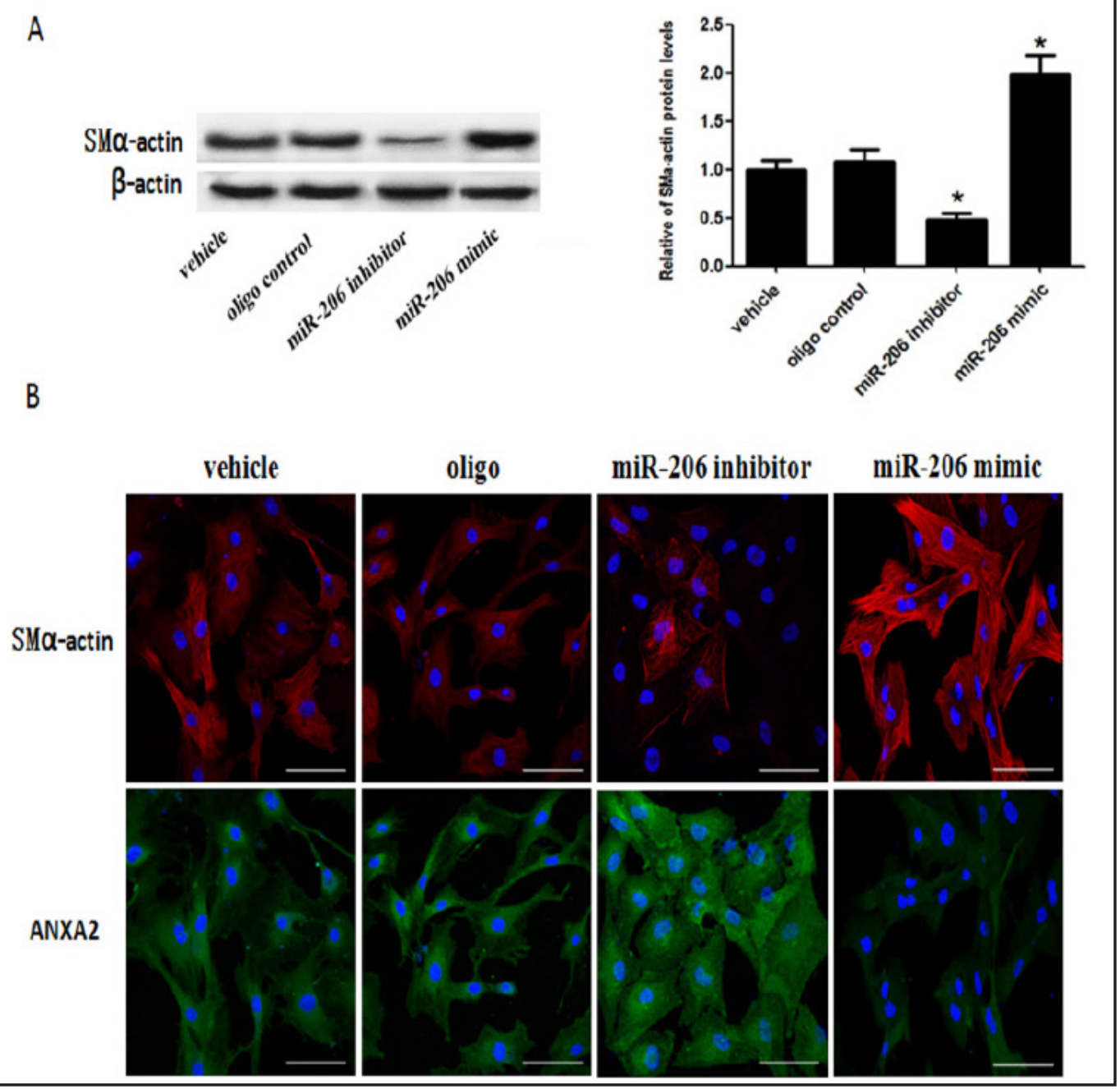

Fig. 2. miR-206 regulates the phenotypic modulation of PASMCs in vitro. (A) Effects of the miR-206 mimic $(10 \mathrm{nM})$ and inhibitor $(200 \mathrm{nM})$ on the expression of SM $\alpha$-actin. Representative western blot bands (left) and mean data generated by densitometry analysis (right). The data shown are the mean $\pm \mathrm{SEM} ; \mathrm{n}=4 .{ }^{*} \mathrm{P}<0.05$ compared with vehicle control.(B) Representative immunofluorescence images of the SM $\alpha$-actin (red) and ANXA2 (green) in VSMCs transfected with the control oligo, miR-206 mimic, or miR-206 inhibitor. Blue represents staining of the nuclei by DAPI. Merged images (right) of SM $\alpha$-actin, ANXA2 and cell nuclei are shown. Scale bar: $100 \mu \mathrm{m}$.

levels of miR-206 with ANXA2 and the differentiation marker protein, SM $\alpha$-actin, in the PASMCs. The PASMCs were treated with 5\% HPS rat serum or normal rat serum for different time periods: $0 \mathrm{~h}, 24 \mathrm{~h}, 48 \mathrm{~h}$ and $72 \mathrm{~h}$. The expression levels of miR-206, ANXA2 and SM -actin were measured at each of these time points. Western blotting showed that the expression levels of the PASMC differentiation marker SM $\alpha$-actin were downregulated, while the expression levels of ANXA2 protein were upregulated in a time-dependent manner in the HPS serum-treated group (Fig. 1A). However, there was no significant change in group C (Fig. 1B). Real-time PCR demonstrated that the pattern of the expression levels of ANXA2 mRNA was similar to that of the protein levels (Fig. 1C and D), whereas the levels of miR-206 showed the opposite expression pattern (Fig. 1E and F). These results indicate that miR-206 may play a role in the phenotypic modulation of the PASMCS by negatively regulating ANXA2 gene expression. 
A

ANXA2

$\beta$-actin
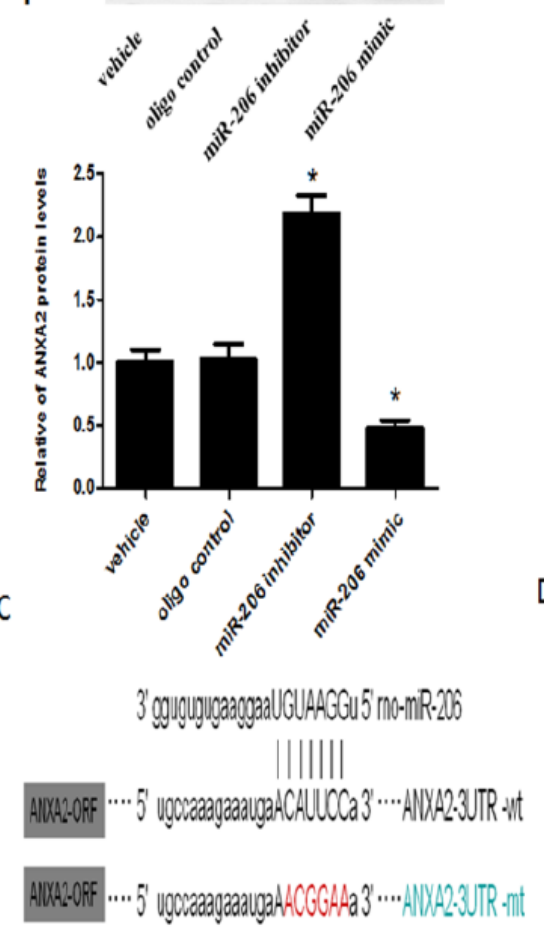

B

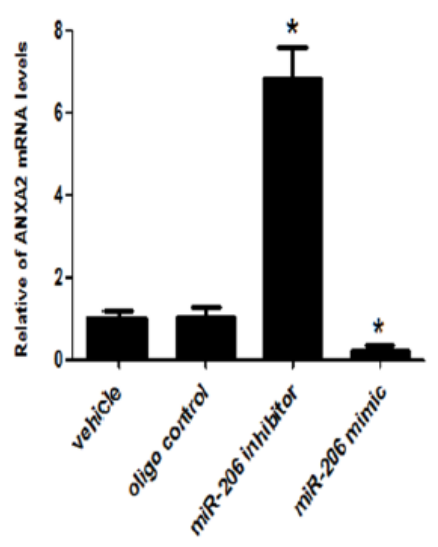

D

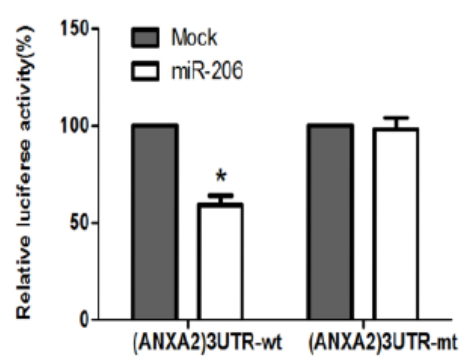

Fig. 3. ANXA2 is a target gene of miR-206 in cultured PASMCs. (A) The miR-206 mimic (10 nM) decreased, whereas the miR-206 inhibitor $(200 \mathrm{nM})$ increased, ANXA2 protein expression in cultured PASMCs. Representative western blot (top) and mean data generated by densitometry analysis (bottom). The data shown are the mean \pm SEM; $n=4 .{ }^{*} \mathrm{P}<0.05$ compared with vehicle control. (B)Effects of miR-206 as indicated by the miR-206 mimic (10 nM) or miR-206 inhibitor ( $200 \mathrm{nM})$ on the upregulation or downregulation of the expression of ANXA2 mRNA. The data shown are the mean $\pm S E M ; n=4 .{ }^{*} \mathrm{P}<0.05$ compared with vehicle control. (C) Mutations of the miR-206 binding sequence in the 3'-UTR of ANXA2. (D) The miR-206 mimic (10 nM) but not the control oligo $(10 \mathrm{nM})$ inhibited the luciferase activity in HEK 293 cells. The inhibitory effect of the miR-206 mimic on the luciferase activity was abrogated in the mutant reporter group. The data shown are the mean \pm SEM; $n=4$. ${ }^{*} \mathrm{P}<0.05$ compared with vehicle control.

miR-206 is involved in the phenotypic modulation of PASMCs

To confirm the effect of miR-206 on the phenotypic modulation of PASMCs, strategies for both gain-of-function and loss-of-function were applied. Either the miR-206 mimic $(10 \mathrm{nM})$ or the miR-206 inhibitor $(200 \mathrm{nM})$ was transiently transfected into the cultured PASMCs in order to positively or negatively regulate the levels of miR-206, respectively. Western blotting was used to detect the expression level of SM $\alpha$-actin at $48 \mathrm{~h}$ after the transient transfection. As expected, the miR-206 mimic upregulated while the miR-206 inhibitor (200 nM) downregulated the protein levels of SM $\alpha$-actin (Fig. 2A). By comparison, the oligo control did not affect the expression of SM $\alpha$-actin in PASMCs (Fig. 2A). The protein expression levels were further confirmed by immunofluorescence using anti-SM -actin and anti-ANXA2 antibodies (Fig. 2B). These results suggest that miR-206 plays an important role in the process of the phenotypic modulation of PASMCs.

miR-206 represses the ANXA2 gene expression by directly targeting the 3'-UTR of ANXA2

We also investigated the influence of miR-206 on the expression of the ANXA2 gene. According to the results of both real-time RT-PCR and western blotting, both the mRNA and 


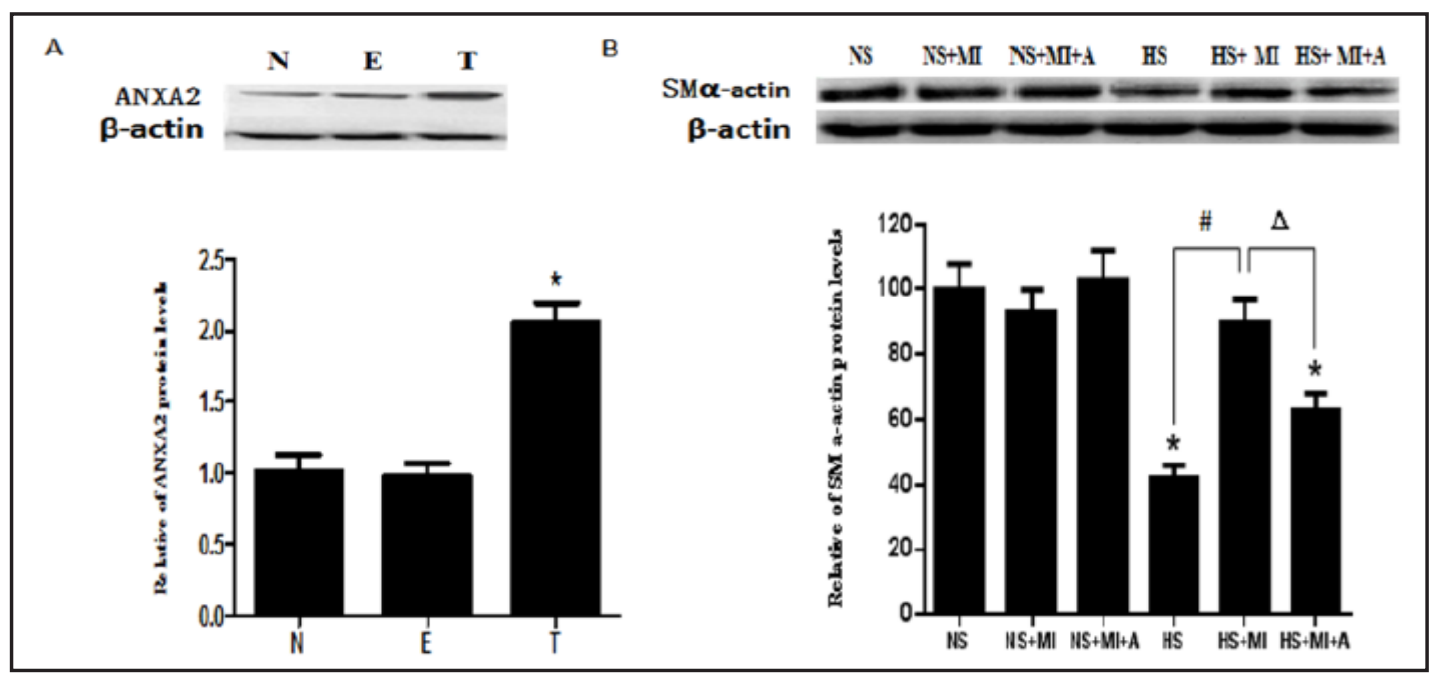

Fig. 4. The miR-206-mediated effect on the phenotypic modulation of the PASMCs is decreased in ANXA2overexpressing cells. (A) The ANXA2 protein expression was significantly increased by transfection with an ANXA2 expression plasmid. Representative western blots (top) and mean data generated by densitometry analysis (bottom) are shown. The data shown are the mean $\pm \mathrm{SEM} ; \mathrm{n}=4 .{ }^{*} \mathrm{P}<0.05$ compared with vehicle control. (B) Effect of the miR-206 mimic (10 nM) on the expression of SM $\alpha$-actin in PASMCs, with or without transfection with an ANXA2 expression plasmid. Representative western blots (top) and mean data generated by densitometry analysis (bottom) are shown. The data shown are the mean $\pm \mathrm{SEM} ; \mathrm{n}=4$. ${ }^{*} \mathrm{P}<0.05$ compared with vehicle control; ${ }^{*} \mathrm{P}<0.05$ compared with HS group; ${ }^{\triangle} \mathrm{P}<0.05$ compared with HS+MI group. N: Non-transfected group; T: transfected group; E: empty vector group. NS: normal rat serum stimulation; NS+MI: miR-206 mimic transfection+normal rat serum stimulation; NS+MI+A: miR-206 mimic and ANXA2 expression plasmid transfection+normal rat serum stimulation; HS: HPS rat serum stimulation; HS+MI: miR-206 mimic transfection+HPS rat serum stimulation; HS+MI+A: miR-206 mimic and ANXA2 expression plasmid transfection+HPS rat serum stimulation.

protein levels of ANXA2 were downregulated by the miR-206 mimic, whereas they were upregulated by the miR-206 inhibitor (Fig. 3A and B). To further confirm that the effect of miR-206 on ANXA2 gene expression was direct and that it was due to the binding of miR-206 to the 3'-UTR of the ANXA2 mRNA, we inserted the ANXA2 3'-UTR region, which contains the predicted miR-206 recognition site (ACAUUCC), into a luciferase reporter plasmid. By cotransfecting the miR-206 mimic and the luciferase vector with the ANXA2 3'-UTR into HEK 293 cells, we demonstrated that the miR-206 mimic significantly repressed the luciferase activity of wild type reporter. This effect was not observed in the analogous mutated reporter in which the sequence of "ACAUUCC" had been replaced with "AACGGAA" to prevent the binding of miR-206 to the ANXA2 3'-UTR region (Fig. 3C and D). These results suggest that miR-206 affects ANXA2 gene expression by directly binding to the complementary 3 '-UTR region of the ANXA2 mRNA.

\section{ANXA2 mediates the effects of miR-206 on the phenotypic modulation and proliferation of PASMCS}

To determine the relative role of ANXA2 in mediating the effects of miR-206 on the phenotypic modulation of PASMCs induced by HPS rat serum, we overexpressed ANXA2 in cultured PASMCs using a transient transfection of the ANXA2-expressing plasmid. Western blotting demonstrated that the expression level of ANXA2 protein was significantly increased at $48 \mathrm{~h}$ after the transient transfection, compared with the vehicle and empty plasmid controls (Fig. 4A). Next, the PASMCs were transfected with the miR-206 mimic (10 nM), the ANXA2-expressing plasmid or both and were stimulated with HPS rat serum for $48 \mathrm{~h}$. The expression levels of SM $\alpha$-actin were measured. Our data showed that the miR-206 mimic, 


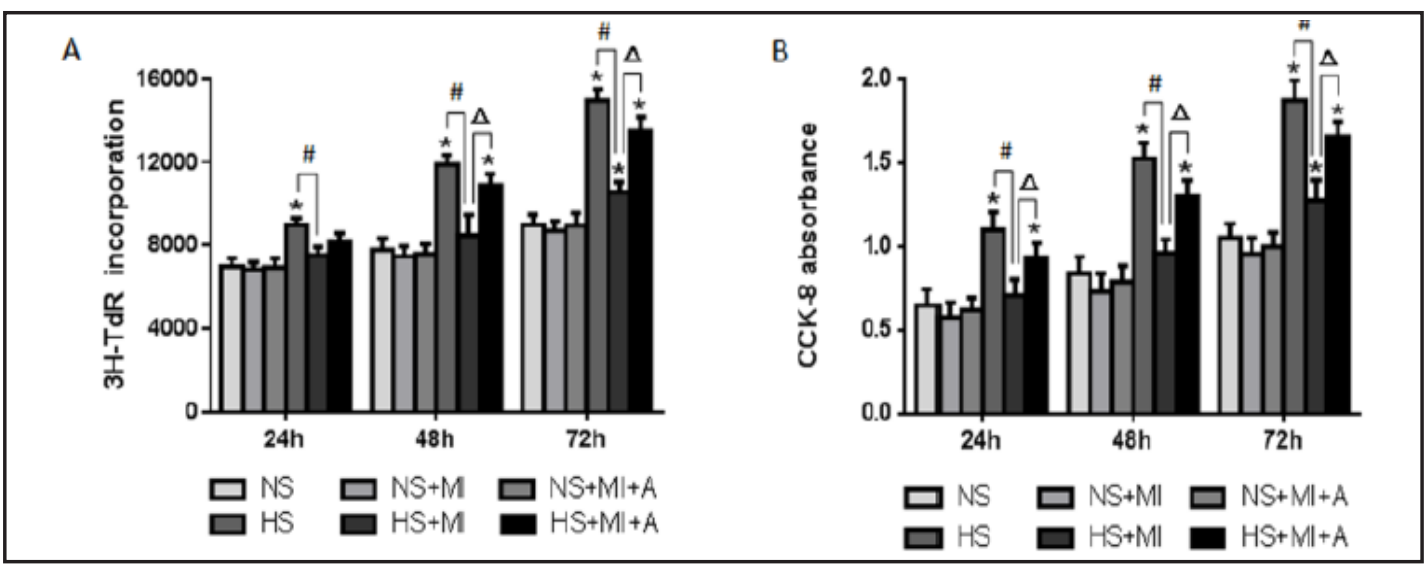

Fig. 5. The miR-206-mediated effect on the proliferation of the PASMCs is decreased in ANXA2-overexpressing cells, as determined by the ${ }^{3} \mathrm{H}-\mathrm{TdR}$ and CCK- 8 assays. (A, B) The changes in the value of the incorporation of ${ }^{3} \mathrm{H}-\mathrm{TdR}$ and the absorbance of CCK-8 assay are displayed. The effect was investigated at $24 \mathrm{~h}, 48 \mathrm{~h}$ and $72 \mathrm{~h}$. The data shown are the mean $\pm \mathrm{SEM} ; \mathrm{n}=4$. ${ }^{*} \mathrm{P}<0.05$ compared with vehicle control; $\mathrm{P}<0.05$ compared with HS group; ${ }^{\triangle} \mathrm{P}<0.05$ compared with HS+MI group. NS: normal rat serum stimulation; HS: HPS rat serum stimulation; HS+MI: miR-206 mimic transfection + HPS rat serum stimulation; HS+MI+A: miR-206 mimic and ANXA2 expression plasmid transfection+ HPS rat serum stimulation.

compared with the vehicle control, effectively blocked the reduced expression of SM $\alpha$-actin mediated by the HPS rat serum. However, co-transfection of the miR-206 mimic and ANXA2expressing plasmid in PASMCs resulted in a significant decrease in SM $\alpha$-actin expression, compared to the group transfected with the miR-206 inhibitor alone (Fig. 4B).

Both the ${ }^{3} \mathrm{H}-\mathrm{TdR}$ incorporation assay and the CCK-8 assay were used to determine the functional role of ANXA2 in mediating the effects of miR-206 on the proliferation of the PASMCs after phenotypic modulation. Our data demonstrated that the miR-206 mimic (10 $\mathrm{nM}$ ) effectively inhibited the HPS rat serum-induced proliferation of PASMCs as indicated by the significant decrease in the incorporation of ${ }^{3} \mathrm{H}-\mathrm{TdR}$ and the absorbance of CCK8 at each time-point, when compared to the control cells. However, this inhibitory effect on proliferation mediated by the miR-206 mimic was abrogated in the PASMCs by the coexpression of the miR-206 mimic and ANXA2. In this case, both the incorporation of ${ }^{3} \mathrm{H}-\mathrm{TdR}$ and the absorbance of CCK-8 were markedly increased at each time-point, compared to the group transfected with the miR-206 mimic alone (Fig. 5A and B).

Taken together, these results suggest that ANXA2 is a functional target gene of miR-206 and that it is involved in the HPS rat serum-induced phenotypic modulation and proliferation of PASMCs.

\section{Discussion}

Due to the stimulation of circulating cytokines and hypoxia, liver cirrhosis and portal hypertension can induce the phenotypic modulation of PASMCs, resulting in PVR. Previous studies of the molecular events underlying PVR have already investigated the signalling pathways, including MLCK, JAK/STAT, PI3K/Akt, and PKC/MAPK, that can regulate the proliferation and migration of PASMCs [19-21]. However, due to the redundant functions among these signalling pathways, the inhibition of a single pathway is usually not effective in preventing the processes that result in PVR. Thus, targeting the upstream events may be a more effective approach to blocking this process. Accumulating evidence has indicated that inhibiting the phenotypic modulation of PASMCs is a key upstream regulatory target because contractile PASMCs cannot proliferate and migrate [22]. Therefore, controlling the phenotypic modulation of PASMCs has become a feasible approach to efficiently prevent PVR and treat pulmonary proliferative vascular diseases. 
ANXA2, which is widely expressed in many tissues and cells including PASMCs, is involved in the regulation of many biological processes, including cell differentiation, proliferation and migration. Specifically, ANXA2 has been shown to participate in the modulation of multiple signal transduction pathways, including p53 signalling, calcium/cAMP/lipid signalling, and focal adhesion signalling $[3,23,24]$. We previously reported that the expression levels of ANXA2 were increased in both the HPS pulmonary artery wall and PASMCs incubated with HPS rat serum, compared with the levels in the control group. Furthermore, the modulation of the ANXA2 gene expression in HPS rat serum-treated PASMCs significantly affects the phenotype and proliferation of the PASMCs. In contrast, the phenotype and proliferation of PASMCs cultured in normal rat serum were not obviously changed [10].

miR-206, a member of the highly conserved miR-206 family, is a functional miRNA in both physiological and pathological conditions. As one of the differentiation-related miRNAs, miR-206 has been demonstrated to play a crucial role in the process of cellular differentiation. Dey et al. [25] showed that the differentiation and proliferation of myoblasts were modulated by miR-206 through its direct target, pax7. Inose et al. [26] identified miR206 as a key regulator of osteoblast differentiation, where it acts by inhibiting the expression of the connexin 43 gene. Jalali et al. [27]confirmed that miR-206 is a potential regulator of proliferation and differentiation of PASMCs in pulmonary arterial hypertension. As an anti-oncogenic miRNA, miR-206 has been shown to inhibit both tumourigenesis and tumour progression. Song et al. [28] determined that miR-206 is a pro-apoptotic activator of cell death that is associated with the regulation of Notch3 function and thus the suppression of tumour growth. Wang et al. [29] found that a low level of miR-206 expression is significantly correlated with the metastatic potential of lung cancer. All these studies indicate that miR206 plays a critical role in cell differentiation and proliferation.

In the present study, we first applied the computational analysis to predict the potential miRNAs that target the 3'-UTR of ANXA2 mRNA, and we identified miR-206 as the most likely regulator. Next, the inverse correlation between miR-206 and ANXA2 and the positive correlation between miR-206 and SM $\alpha$-actin were confirmed in cultured PASMCs treated with HPS rat serum or normal rat serum. Based on this observation, we confirmed that miR206 downregulates ANXA2 and up-regulates SM $\alpha$-actin gene expression through gain-offunction and loss-of-function approaches. Consistent with the decrease in the expression of ANXA2 induced by miR-206, the ability of miR-206 to bind to the 3'-UTR of ANXA2 mRNA was validated using a dual luciferase reporter gene assay. Finally, we confirmed that the phenotypic modulation of PAMSCs mediated by ANXA2 is regulated by the HPS rat seruminduced inhibition of miR-206 expression.

Taken together, our study for the first time revealed that miR-206 acts as a novel regulator of the PASMC phenotype via its target gene ANXA2. The decreased miR-206 levels increased the expression of ANXA2, while increases in the expression of ANXA2 suppressed the PASMC phenotype marker gene, possibly via downstream molecules such as ERK1/2, NF- $\kappa$ B and $\beta$-catenin $[30,31]$. The dedifferentiated PASMCs demonstrated increased proliferation rates, resulting in PVR [32]. Although our current study was limited by a lack of animal and human tissue experiments, the findings may still have a significant impact on our understanding of HPS-associated PVR.

\section{Disclosure Statement}

All authors declare there are no conflicts of interest.

\section{Acknowledgments}

This work is supported by grant nos. 30700347, 30872448, 81170053 and 81170414 from the National Science Foundation of China (NSFC). 


\section{Cellular Physiology and Biochemistry}

\section{References}

1 Ho V: Current concepts in the management of hepatopulmonary syndrome. Vasc Health Risk Manag 2008;4:1035.

2 Chan MC, Weisman AS, Kang H, Nguyen PH, Hickman T, Mecker SV, Hill NS, Lagna G, Hata A: The amiloride derivative phenamil attenuates pulmonary vascular remodeling by activating NFAT and the bone morphogenetic protein signaling pathway. Mol Cell Biol 2011;31:517-530.

3 Wang C-Y, Chen C-L, Tseng Y-L, Fang Y-T, Lin Y-S, Su W-C, Chen C-C, Chang K-C, Wang Y-C, Lin C-F: Annexin A2 silencing induces G2 arrest of non-small cell lung cancer cells through p53-dependent and-independent mechanisms. J Biol Chem 2012;287:32512-32524.

4 Gerke V, Creutz CE, Moss SE: Annexins: linking Ca2+ signalling to membrane dynamics. Nat Rev Mol Cell Biol 2005;6:449-461. Gerke V, Moss SE: Annexins: from structure to function. Physiol Rev 2002;82:331-371.

Domon M, Nasir MN, Matar G, Pikula S, Besson F, Bandorowicz-Pikula J: Annexins as organizers of cholesterol-and sphingomyelin-enriched membrane microdomains in Niemann-Pick type C disease. Cell Mol Life Sci 2012;69:1773-1785.

7 Mohammad HS, Kurokohchi K, Yoneyama H, Tokuda M, Morishita A, Jian G, Shi L, Murota M, Tani J, Kato K: Annexin A2 expression and phosphorylation are up-regulated in hepatocellular carcinoma. Int J Oncol 2008;33:1157.

8 Deng S, Wang J, Hou L, Li J, Chen G, Jing B, Zhang X, Yang Z: Annexin A1, A2, A4 and A5 play important roles in breast cancer, pancreatic cancer and laryngeal carcinoma, alone and/or synergistically. Oncol Lett 2013;5:107-112.

9 Wang CY, Lin CF: Annexin A2 facilitates cell cycle by sustaining c-Jun N-terminal kinase-inhibited p53 expression in lung adenocarcinoma. Mol Biol Cell 2011, 22.

10 Zeng J, Yi B, Wang Z, Ning J, Wang X, Lu K: Effect of annexin A2 on hepatopulmonary syndrome rat seruminduced proliferation of pulmonary arterial smooth muscle cells. Respir Physiol Neurobiol 2013;185:332338.

11 Huang B, Deora AB, He K-L, Chen K, Sui G, Jacovina AT, Almeida D, Hong P, Burgman P, Hajjar KA: Hypoxiainducible factor-1 drives annexin A2 system-mediated perivascular fibrin clearance in oxygen-induced retinopathy in mice. Blood 2011;118:2918-2929.

12 Wu M-H, Chuang P-C, Lin Y-J, Tsai S-J: Suppression of annexin A2 by prostaglandin E2 impairs phagocytic ability of peritoneal macrophages in women with endometriosis. Hum Reprod 2013;28:1045-1053.

13 Winter J, Jung S, Keller S, Gregory RI, Diederichs S: Many roads to maturity: microRNA biogenesis pathways and their regulation. Nat Cell Biol 2009;11:228-234.

14 Caruso P, MacLean MR, Khanin R, McClure J, Soon E, Southgate M, MacDonald RA, Greig JA, Robertson $\mathrm{KE}$, Masson R: Dynamic changes in lung microRNA profiles during the development of pulmonary hypertension due to chronic hypoxia and monocrotaline. Arterioscler Thromb Vasc Biol 2010;30:716-723.

15 Yi B, Lu JY, Bai L, Wang GS, Qian GS: Adenovirus-mediated protein-kinase-GIalpha suppresses the hypoxiainduced proliferation and phenotype-switching of pulmonary arterial smooth muscle cell. Zhonghua Nei Ke Za Zhi 2010;49:385-388.

16 Yi B, Cui J, Ning J-n, Wang G-s, Qian G-s, Lu K-z: Over-expression of PKGI $\alpha$ inhibits hypoxia-induced proliferation, Akt activation, and phenotype modulation of human PASMCs: The role of phenotype modulation of PASMCs in pulmonary vascular remodeling. Gene 2012;492:354-360.

-17 Yi B, Cui J, Ning J, Gu J, Wang G, Bai L, Qian G, Lu K: cGMP-dependent protein kinase I $\alpha$ transfection inhibits hypoxia-induced migration, phenotype modulation and annexins A1 expression in human pulmonary artery smooth muscle cells. Biochem Biophys Res Commun 2012;418:598-602.

18 Chen Y, Yi B, Wang Z, Gu J, Li Y, Cui J, Lu K: Paxillin suppresses the proliferation of HPS rat serum treated PASMCs by up-regulating the expression of cytoskeletal proteins. Mol Biosyst 2014;10:759-766.

19 Bai L, Yu Z, Qian G, Qian P, Jiang J, Wang G, Bai C: SOCS3 was induced by hypoxia and suppressed STAT3 phosphorylation in pulmonary arterial smooth muscle cells. Respir Physiol Neurobiol 2006;152:83-91.

20 Luo C, Yi B, Bai L, Xia Y, Wang G, Qian G, Feng H: Suppression of Akt1 phosphorylation by adenoviral transfer of the PTEN gene inhibits hypoxia-induced proliferation of rat pulmonary arterial smooth muscle cells. Biochem Biophys Res Commun 2010;397:486-492. 
Chen et al.: miR-206 Controls Phenotypic Modulation of PASMCs

21 Wang G-S, Qian G-S, Zhou D-S, Zhao J-Q: JAK-STAT signaling pathway in pulmonary arterial smooth muscle cells is activated by hypoxia. Cell Biol Int 2005;29:598-603.

22 Kouri FM, Queisser MA, Königshoff M, Chrobak I, Preissner KT, Seeger W, Eickelberg O: Plasminogen activator inhibitor type 1 inhibits smooth muscle cell proliferation in pulmonary arterial hypertension. Int J Biochem Cell Biol 2008;40:1872-1882.

23 Brandherm I, Disse J, Zeuschner D, Gerke V: cAMP-induced secretion of endothelial von Willebrand factor is regulated by a phosphorylation/dephosphorylation switch in annexin A2. Blood 2013;122:1042-1051.

-24 Hayes MJ, Moss SE: Annexin 2 has a dual role as regulator and effector of v-Src in cell transformation. J Biol Chem 2009;284:10202-10210.

-25 Dey BK, Gagan J, Dutta A: miR-206 and-486 induce myoblast differentiation by downregulating Pax7. Mol Cell Biol 2011;31:203-214.

-26 Anderson C, Catoe H, Werner R: MIR-206 regulates connexin43 expression during skeletal muscle development. Nucleic Acids Res 2006;34:5863-5871.

27 Jalali S, Ramanathan GK, Parthasarathy PT, Aljubran S, Galam L, Yunus A, Garcia S, Cox RR Jr, Lockey RF, Kolliputi N: Mir-206 regulates pulmonary artery smooth muscle cell proliferation and differentiation. PLoS One 2012; 7:e46808.

28 Song G, Zhang Y, Wang L: MicroRNA-206 targets notch3, activates apoptosis, and inhibits tumor cell migration and focus formation. J Biol Chem 2009;284:31921-31927.

29 Wang X, Ling C, Bai Y, Zhao J: MicroRNA-206 Is Associated With Invasion and Metastasis of Lung Cancer. Anat Rec 2011;294:88-92.

-30 Garrido-Gómez T, Dominguez F, Quiñonero A, Estella C, Vilella F, Pellicer A, Simon C: Annexin A2 is critical for embryo adhesiveness to the human endometrium by RhoA activation through F-actin regulation. FASEB J 2012;26:3715-3727.

-31 Sarkar S, Swiercz R, Kantara C, Hajjar KA, Singh P: Annexin A2 mediates up-regulation of NF-кB, $\beta$-catenin, and stem cell in response to progastrin in mice and HEK-293 cells. Gastroenterology 2011;140:583-595. e584.

32 Yeager ME, Frid MG, Stenmark KR: Progenitor cells in pulmonary vascular remodeling. Pulm Circ 2011;1:316. 